\title{
Incompressibility of finite fermionic systems: Stable and exotic atomic nuclei
}

\author{
E. Khan, ${ }^{1}$ N. Paar, ${ }^{2}$ D. Vretenar, ${ }^{2}$ Li-Gang Cao, ${ }^{3}$ H. Sagawa,,${ }^{4,5}$ and G. Colò ${ }^{6}$ \\ ${ }^{1}$ Institut de Physique Nucléaire, Université Paris-Sud, IN2P3-CNRS, F-91406 Orsay Cedex, France \\ ${ }^{2}$ Physics Department, Faculty of Science, University of Zagreb, 10000 Zagreb, Croatia \\ ${ }^{3}$ Institute of Modern Physics, Chinese Academy of Science, Lanzhou 730000, China \\ ${ }^{4}$ Center for Mathematics and Physics, University of Aizu, Aizu-Wakamatsu, 965-8580 Fukushima, Japan \\ ${ }^{5}$ RIKEN Nishina Center, Wako 351-0198, Japan \\ ${ }^{6}$ Dipartimento di Fisica, Università degli Studi and INFN Sezione di Milano, Via Celoria 16, 20133 Milano, Italy
}

(Received 26 April 2013; published 20 June 2013)

\begin{abstract}
The incompressibility of finite fermionic systems is investigated using analytical approaches and microscopic models. The incompressibility of a system is directly linked to the zero-point kinetic energy of constituent fermions, and this is a universal feature of fermionic systems. In the case of atomic nuclei, this implies a constant value of the incompressibility in medium-heavy and heavy nuclei. The evolution of nuclear incompressibility along $\mathrm{Sn}$ and $\mathrm{Pb}$ isotopic chains is analyzed using global microscopic models, based on both nonrelativistic and relativistic energy functionals. The result is an almost constant incompressibility in stable nuclei and systems not far from stability and a steep decrease in nuclei with pronounced neutron excess, caused by the emergence of a soft monopole mode in neutron-rich nuclei.
\end{abstract}

DOI: 10.1103/PhysRevC.87.064311

PACS number(s): 21.10.Re, 21.65.-f, 21.60.Jz

\section{INTRODUCTION}

Recent advances in experimental and theoretical investigations of nuclear incompressibility have also enabled studies of the evolution of incompressibility in isotopic chains [1-6]. As for many other structure phenomena, for instance the evolution of magic numbers [7], a study of the isotopic dependence might provide a more general and deeper understanding of nuclear incompressibility. It has recently been inferred that measurement of isoscalar giant monopole resonances (ISGMRs) may probe nuclear incompressibility in a range of low nucleonic densities, rather than exactly at saturation density [8]. Experiments on isotopic chains [2,3], possibly also extended to exotic nuclei $[9,10]$, are therefore important for constraining the density dependence of the incompressibility. Investigations of isotopic chains also enable studies of pairing and shell effects on incompressibility. It has been shown that one cannot neglect the effect of shell closure on nuclear incompressibility, and pairing correlations affect the position of the GMR excitation energy [4,5]. The effect of shell closure (and therefore magicity) on incompressibility has been discussed in Ref. [11]. However, there is a general consensus that pairing effects alone are not large enough to explain the available data $[6,12,13]$. Early microscopic studies of nuclear incompressibility were carried out using Skyrme effective forces. Later, with the inclusion of relativistic mean-field models, detailed comparisons among different model predictions led to the conclusion that the nuclear matter incompressibility extracted from, e.g., ${ }^{208} \mathrm{~Pb}$ lies in the range $\approx 240 \pm 20 \mathrm{MeV}$ (cf. [14] and references therein). The remaining uncertainty reflects our incomplete understanding of the specific effects related to finite nuclei and further justifies the need of studying extended isotopic chains.

The theoretical framework of energy density functionals (EDFs) $[15,16]$ is the tool of choice for a quantitative analysis of these phenomena. Preliminary studies, undertaken in the 1980s, analyzed the dependence of the nuclear incompressibility on mass number, but only four doubly magic nuclei were considered and it was suggested that nuclear incompressibility is rather constant for heavy nuclei $[17,18]$. Another aspect is that the occurrence of low-energy monopole strength in exotic nuclei has recently been predicted [19-21], and this might affect nuclear incompressibility. More generally, the present study of extended isotopic chains could also be useful for understanding incompressibility in other fermionic systems, or for astrophysical processes such as core-collapse supernovae. The use of both analytical and fully microscopic approaches will provide complementary information about the incompressibility in both stable and exotic nuclei.

Section II defines the analytical relations between incompressibility and other relevant nuclear quantities. Section III compares the analytical results with the microscopic ones obtained using the EDF framework. A brief summary and conclusions are given in Sec. IV.

\section{INCOMPRESSIBILITY OF A FINITE FERMIONIC SYSTEM}

In this section we review the relations between the nuclear incompressibility $K_{A}$ and the nucleonic kinetic energy in the following analytical models: infinite nuclear matter, Fermi gas, and the harmonic oscillator. For instance, in the free Fermi gas approach the incompressibility of the system is directly linked to the Fermi energy [22].

\section{A. Definitions}

The incompressibility of a fermionic system composed of $A$ particles is defined as the second derivative (curvature) of its energy with respect to the mean square radius at the minimum [17]:

$$
\left.K_{A} \equiv 4\left\langle r^{2}\right\rangle \frac{d^{2} E / A}{d\left\langle r^{2}\right\rangle^{2}}\right|_{\text {g.s. }}
$$


where the label g.s. indicates that the quantities are evaluated in the ground state. For the isoscalar monopole transition operator

$$
\hat{Q}=\sum_{i=1}^{A} r_{i}^{2}
$$

the dielectric theorem $[17,18,20]$ yields

$$
K_{A}=\frac{2 A\left\langle r^{2}\right\rangle_{\mathrm{g} . \mathrm{s} .}^{2}}{m_{-1}}
$$

where $m_{-1}$ is the inverse energy weighted sum rule, proportional to the polarizability of the system.

The energy of the isoscalar GMR can be defined by the following relation $[17,18]$ :

$$
E_{\mathrm{GMR}}=\sqrt{\frac{m_{1}}{m_{-1}}} .
$$

The moment $m_{1}$ is evaluated by the double commutator using the Thouless theorem [23]:

$$
m_{1}=\frac{2 \hbar^{2} A}{m}\left\langle r^{2}\right\rangle_{\text {g.s. }}
$$

where $m$ is the fermion mass, that is, $m c^{2} \approx 938 \mathrm{MeV}$ for the nucleon. Equations (3)-(5) lead to [17]

$$
E_{\mathrm{GMR}}=\sqrt{\frac{\hbar^{2} K_{A}}{m\left\langle r^{2}\right\rangle}} .
$$

\section{B. An analytical study}

An analytical derivation of the nuclear incompressibility $K_{A}$ in the nuclear matter, the Fermi gas, and the harmonic oscillator models will provide a complementary insight into the microscopic results of the next section.

\section{Nuclear matter and the Fermi gas}

Let us first consider infinite homogeneous nuclear matter. The nuclear matter incompressibility at saturation $K_{\infty}$ is understood fairly well, whereas the behavior of the, more general, density-dependent incompressibility has not been studied much. The general density-dependent incompressibility $K(\rho)$ is derived from the thermodynamical compressibility $\chi(\rho)$ as $[8,24]$

$$
K(\rho) \equiv \frac{9}{\rho \chi(\rho)}=9 \rho^{2} \frac{\partial^{2} E(\rho) / A}{\partial \rho^{2}}+\frac{18}{\rho} P(\rho),
$$

where the pressure is

$$
P(\rho) \equiv \rho^{2} \frac{\partial E(\rho) / A}{\partial \rho} .
$$

Neglecting internucleon interactions, one finds $[17,22]$

$$
K(\rho)=6 \epsilon_{F}=\frac{6 \hbar^{2}}{2 m}\left(\frac{3 \pi^{2}}{2}\right)^{2 / 3} \rho^{2 / 3} .
$$

This equation exhibits the density dependence of the incompressibility, known to play an important role in the relation between the measurement of GMRs and the equation of state [8]: in the case of noninteracting nuclear matter the density-dependent incompressibility is proportional to $\rho^{2 / 3}$. At saturation density $\left(\rho_{0}=0.16 \mathrm{fm}^{-3}\right)$ Eq. (9) yields $K\left(\rho_{0}\right)=$ $220 \mathrm{MeV}$, and at the so-called crossing density [8] $\left(\rho_{c}=\right.$ $0.1 \mathrm{fm}^{-3}$ ) one gets $K\left(\rho_{c}\right)=160 \mathrm{MeV}$. This shows that the density dependence of the incompressibility of noninteracting nuclear matter presents a good approximation at saturation density but not at the crossing density, for which $K_{c} \simeq 40 \mathrm{MeV}$ is the expected value [8]. This can be understood because at the crossing density interaction effects are expected to be more important, whereas at saturation density the homogeneous nucleonic system can simply be modeled by noninteracting hard spheres. Considering an average spacing $r_{0}$ among the constituents, one gets for the homogeneous density of the system

$$
\rho=\left(\frac{4}{3} \pi r_{0}^{3}\right)^{-1} .
$$

In the case of nucleons, $r_{0} \approx 1.2 \mathrm{fm}$ yields $\rho_{0} \approx 0.15 \mathrm{fm}^{-3}$, and this shows the validity of the model in the vicinity of saturation density.

Approximating the nucleus by a free Fermi gas of nucleons, we study a spherical fermionic $A$-body system with a typical equilibrium internucleon distance $r_{0}$. The radius of the system is then $R=r_{0} A^{1 / 3}$. To establish a link between the incompressibility $K_{A}$ and the zero-point energy, one inserts Eq. (10) in Eq. (9), and this leads to

$$
K_{A}=\frac{3}{4}(9 \pi)^{2 / 3} T_{0} \approx 7 T_{0},
$$

where $T_{0}$ is the zero-point kinetic energy (ZPE) of a fermion in the system [25]:

$$
T_{0} \equiv \frac{\hbar^{2}}{m r_{0}^{2}}
$$

The ZPE, first discussed by Mottelson [25], depends only on the characteristics of the constituent fermions, but not on the system itself. For nucleons $T_{0} \approx 30 \mathrm{MeV}$, and this yields $K_{A} \approx 210 \mathrm{MeV}$. It should be noted that Blaizot also derived a relation between $K_{A}$ and the average kinetic energy [17]. However, the average kinetic energy depends on the model and the system considered. Here the incompressibility is linked to the ZPE, which does not depend on any model nor on the specific system. Equation (11) shows that the larger the ZPE, the more difficult it becomes to compress the system: quantum localization of individual nucleons determines the nuclear incompressibility. As a consequence, $K_{A}$ should be independent of $A$ because the incompressibility is directly linked to the nucleonic ZPE (provided, of course, that the surface to volume ratio is small enough).

\section{Harmonic oscillator}

In the harmonic oscillator (HO) approximation the $L=0$ radial compression mode (GMR) can be represented by a $2 \hbar \omega$ mode [26], where $\hbar \omega$ is

$$
\hbar \omega \approx \frac{5}{4}\left(\frac{3}{2}\right)^{1 / 3} \frac{\hbar^{2}}{m r_{0}^{2}} A^{-1 / 3} .
$$


By assuming spherical density, $\left\langle r^{2}\right\rangle$ in Eq. (6) can be approximated by $3 R^{2} / 5$, where $R$ is the nuclear radius, and the GMR energy becomes

$$
E_{\mathrm{GMR}}=\sqrt{\frac{5 \hbar^{2} K_{A}}{3 m r_{0}^{2}}} A^{-1 / 3} .
$$

In the $\mathrm{HO}$ approximation $E_{\mathrm{GMR}}=2 \hbar \omega$, and from Eqs. (13) and (14) one obtains

$$
K_{A} \approx \frac{15}{4}\left(\frac{3}{2}\right)^{2 / 3} \frac{\hbar^{2}}{m r_{0}^{2}} \approx 5 T_{0}
$$

Therefore, in the HO approximation the compression modulus is also found to be constant and directly related to the ZPE. This again means that the higher the ZPE, the harder it becomes to compress the system. For nuclei $K_{A}$ is expected to be a constant in the lowest-order approximation, depending only on the nucleon mass and the typical internucleon distance. Equation (15) yields $K_{A} \simeq 140 \mathrm{MeV}$, and one expects that the $\mathrm{HO}$ value is more accurate than the one obtained in the Fermi gas model. This is because the HO potential presents a much better first-order approximation to the nuclear mean-field potential.

If independent of $A$, however, the value $K_{A} \approx 140 \mathrm{MeV}$ does not correspond to the limit of infinite nuclear matter when $A \rightarrow \infty$, that is, $K_{\infty}=220 \mathrm{MeV}$. The reason is that the $\mathrm{HO}$ value has been derived under the assumption that the GMR is a $2 \hbar \omega$ mode, but this is not valid any more for infinite nuclear matter. In that case, it is the incompressibility of the Fermi gas that should be used, yielding $K_{A}=210 \mathrm{MeV}$ as described above. This difference is also confirmed by the fact that (i) $K_{A} \approx 140 \mathrm{MeV}$ is predicted by microscopic calculations in finite nuclei [5,6] and (ii) $K_{\infty} \geqslant 200 \mathrm{MeV}$ is obtained by microscopic extrapolation of GMR measurements to infinite nuclear matter [8].

\section{MICROSCOPIC CALCULATION OF THE NUCLEAR INCOMPRESSIBILITY IN ISOTOPIC CHAINS}

The first fully microscopic studies of the behavior of $K_{A}$ as a function of the number of nucleons were performed in the 1980s $[17,18]$. At that time only four spherical magic nuclei were considered: ${ }^{16} \mathrm{O},{ }^{40} \mathrm{Ca},{ }^{90} \mathrm{Zr}$, and ${ }^{208} \mathrm{~Pb}$. It was inferred that $K_{A}$ is not very sensitive to $A$, although a general conclusion was hindered by the small number of nuclei included in the analysis. One should also emphasize that light nuclei are unlikely to fit into the general picture because of the large surface to volume ratio. In light nuclei the experimental ISGMR strength distribution displays pronounced fragmentation, and it is hard to extract a well-defined value for the monopole energy.

To investigate the evolution of nuclear incompressibility along isotopic chains, it is necessary to use global, EDFbased models, which also take into account pairing effects. The constrained Hartree-Fock-Bogoliubov (CHFB) and the quasiparticle random-phase approximation (QRPA) [27] are employed in the present study. For the latter, both relativistic and nonrelativistic implementations will be used to reduce possible model dependencies. Our aim is to verify, in a microscopic calculation, the constant behavior of $K_{A}$ predicted by the analytical model described in the previous section.

\section{A. Formalism}

Microscopic EDF-based calculations of isoscalar GMRs are usually performed using the CHFB or the QRPA framework [27]. In this study we calculate the GMR excitation energies using the CHFB approach based on a Skyrme EDF, and QRPA calculations are carried out using both Skyrme and relativistic functionals.

\section{Constrained $\mathrm{HFB}$}

The extension of the constrained Hartree-Fock (HF) method [18] to include pairing correlations, that is, the CHFB approach, has been detailed in Ref. [20] and has been applied also in [5]. In the CHFB method the energy of the GMR is computed using Eq. (4), with the $m_{1}$ moment determined by the HFB r.m.s. radius [Eq. (5)]. To evaluate the $m_{-1}$ moment, the constraint associated with the isoscalar monopole operator is added to the HFB Hamiltonian

$$
\hat{H}_{\text {constr. }}=\hat{H}+\lambda \hat{Q} .
$$

The $m_{-1}$ moment is obtained from the derivative of the expectation value of the monopole operator in the CHFB eigenstate $|\lambda\rangle$, using the dielectric theorem

$$
m_{-1}=-\frac{1}{2}\left[\frac{\partial}{\partial \lambda}\langle\lambda|\hat{Q}| \lambda\rangle\right]_{\lambda=0} .
$$

In the present calculation we employ the Skyrme functional SLy5 [28], together with the density-dependent delta-pairing interaction

$$
V_{\text {pair }}=V_{0}\left[1-\eta\left(\frac{\rho(r)}{\rho_{0}}\right)^{\alpha}\right] \delta\left(\mathbf{r}_{1}-\mathbf{r}_{2}\right),
$$

with $V_{0}=-285 \mathrm{MeV} \mathrm{fm}{ }^{3}, \eta=0.35, \alpha=1$, and $\rho_{0}=$ $0.16 \mathrm{fm}^{-3}$. This pairing interaction has been designed to reproduce the experimental two-neutron separation energies for the even-even isotopes ${ }^{114-134} \mathrm{Sn}$ [29]. The energy cutoff (quasiparticle energy) is $E_{\max }=60 \mathrm{MeV}$, and $j_{\max }=15 / 2$.

\section{Quasiparticle random phase approximation}

For completeness we briefly outline the details of the QRPA calculations. The Skyrme HF-BCS equations for the ground state are solved in coordinate space. The radial mesh extends to $18 \mathrm{fm}$, with a spacing of $0.1 \mathrm{fm}$. For all nuclei analyzed in the present study we have verified the stability of the results with the variation of the size of the mesh. Pairing correlations are taken into account by a contact volume-pairing interaction, with strength $V_{0}=-218(-265) \mathrm{MeV} \mathrm{fm}^{3}$ for $\mathrm{Sn}$ $(\mathrm{Pb})$ isotopes. The pairing strength is adjusted to reproduce the empirical pairing gaps extracted from data on odd-even mass differences (five-point formula). The single-particle continuum is discretized by placing nuclei in a spherical box 
of radius $=18 \mathrm{fm}$. For each set of spherical single-particle quantum numbers $(l, j)$, the QRPA model space includes unoccupied states up to the maximum number of nodes given by $n_{\max }=n_{\text {last }}+12$, where $n_{\text {last }}$ is the number of nodes for the last occupied state with a given $(l, j)$. The convergence of the results has been verified by calculating the strength function of the ISGMR. For a state with good angular momentum and parity $J^{\pi}$, the QRPA matrix equation reads

$$
\left(\begin{array}{cc}
A & B \\
B^{*} & A^{*}
\end{array}\right)\left(\begin{array}{l}
X^{n} \\
Y^{n}
\end{array}\right)=\hbar \omega_{n}\left(\begin{array}{cc}
1 & 0 \\
0 & -1
\end{array}\right)\left(\begin{array}{l}
X^{n} \\
Y^{n}
\end{array}\right),
$$

where $\hbar \omega_{n}$ is the energy of the $n$th QRPA state. $X^{n}$ and $Y^{n}$ are the corresponding forward and backward amplitudes, respectively. The explicit expressions for the matrices $A$ and $B$ are given, for instance, in Refs. [30,31]. The particle-hole (p-h) matrix elements are computed from the Skyrme energy density functional including all terms, that is, also the two-body spin-orbit and two-body Coulomb interactions.

The resulting moments of the QRPA strength distribution are calculated as

$$
m_{k}=\int E^{k} S(E) d E,
$$

where $S(E)=\sum_{n}|\langle 0|\hat{Q}| n\rangle|^{2} \delta\left(E-E_{n}\right)$ is the strength function associated with the monopole operator defined in Eq. (2).

In the present analysis we also employ the QRPA based on relativistic nuclear energy density functionals. More details about this framework are given in Refs. [16,32,33]. It is realized in terms of the fully self-consistent RQRPA based on the relativistic Hartree-Bogoliubov model (RHB). The RQRPA equations are formulated in the canonical single-nucleon basis of the RHB model, by employing effective Lagrangians with density-dependent meson-nucleon couplings [32] in the particle-hole channel and the finite-range Gogny interaction [34] in the pairing channel. The present calculation of the isoscalar monopole response is carried out using the density-dependent meson-exchange effective Lagrangian DD-ME2 [35].

\section{B. Results and interpretation}

The values of nuclear incompressibility calculated using the CHFB, nonrelativistic QRPA, and relativistic QRPA for the chains of $\mathrm{Sn}$ and $\mathrm{Pb}$ isotopes are displayed in Fig. 1. With the GMR excitation energies $E_{\mathrm{GMR}}$ determined by the moments of the strength distributions [cf. Eq. (4)], $K_{A}$ is calculated from Eq. (6). We note that the three microscopic models predict remarkably similar isotopic trends, with values of $K_{A}$ for individual nuclei that do not differ by more than $10 \%$. The nuclear incompressibility $K_{A}$ is rather constant for stable nuclei and systems with moderate neutron excess, but it displays a sharp decrease beyond the neutron shells $N=82$ for $\mathrm{Sn}$ and $N=126$ for $\mathrm{Pb}$ isotopes. This behavior of $K_{A}$ was already observed in Ref. [6], in which the isotopic $\mathrm{Pb}$ and Sn chains were calculated in the QRPA with both the SLy4 and UNEDF0 effective interactions. The rather constant values of $K_{A}$ shown in Fig. 1 are close to $140 \mathrm{MeV}$, in quantitative agreement with the HO prediction [Eq. (15)].

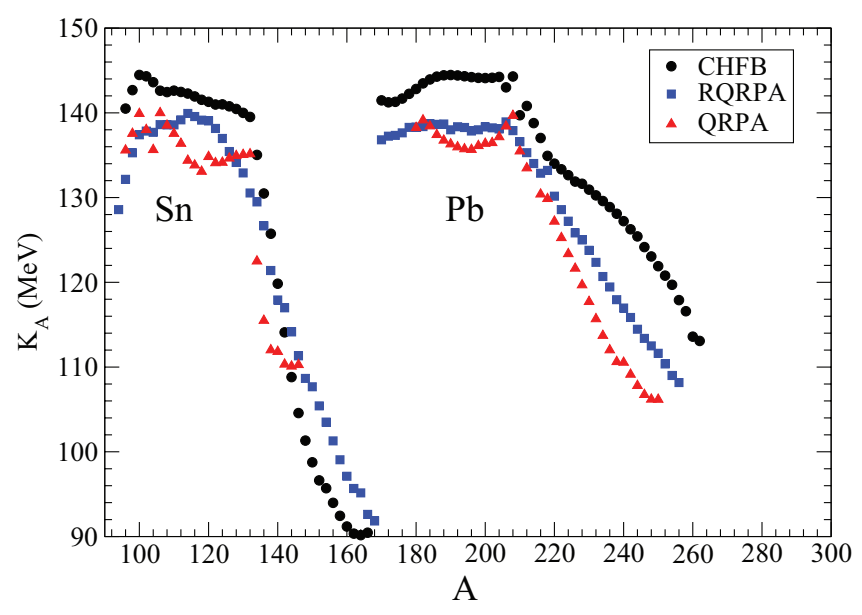

FIG. 1. (Color online) Nuclear incompressibility of the Sn and $\mathrm{Pb}$ isotopic chains, calculated using the microscopic Skyrme-CHFB method (circles), the Skyrme-QRPA (triangles), and the relativistic QRPA (squares).

This shows that the analytical approach of Sec. II B 2 provides an accurate approximation for $K_{A}$ in isotopes close to stability, for which valence neutrons typically occupy the same major shell. In the model of Sec. II, the energy of the monopole is taken as $2 \hbar \omega$, in accordance with the experimental trend $(\hbar \omega \approx$ $41 A^{-1 / 3}$ and $E_{\mathrm{ISGMR}} \approx 80 A^{-1 / 3}$ ). Many energy functionals are characterized by values of the effective mass $m^{*} / m$ that are smaller than 1. In this case it is the RPA attractive residual interaction that lowers the monopole energy to its empirical value, even when the unperturbed energy is larger than $2 \hbar \omega$, that is, $\left(2 \hbar \omega m / m^{*}\right)$. Therefore, one understands that the HO estimate of $K_{A} \simeq 140 \mathrm{MeV}$ is close to the results obtained from EDF calculations. Even though the latter include effects of the effective mass and the residual interaction, there is an approximate cancellation of these effects.

The sudden decrease of $K_{A}$ beyond the doubly magic nuclei ${ }^{132} \mathrm{Sn}$ and ${ }^{208} \mathrm{~Pb}$ is, of course, related to the opening of new major shells in which monopole particle-hole configurations are formed. This leads to the appearance of a soft monopole mode that increases the monopole moment $m_{-1}$ and, consequently, decreases the deduced value of $K_{A}$. Soft monopole modes have been predicted in several studies [19-21]. In Ref. [20] the effect of a soft monopole mode on the excitation energy of the GMR was analyzed for $\mathrm{Ni}$ and $\mathrm{Ca}$ isotopes using the Skyrme-QRPA model, and similar conclusions were reached. Figures 2 and 3 display the isoscalar monopole response in a number of $\mathrm{Sn}$ and $\mathrm{Pb}$ isotopes, calculated using both the nonrelativistic and relativistic QRPA with the functionals SLy5 and DD-ME2, respectively. The emergence of a soft monopole mode for exotic nuclei far from stability is clearly visible and can be related to the decrease of the nuclear incompressibility $K_{A}$ in neutron-rich nuclei.

The steep decrease of $K_{A}$ shown in Fig. 1 raises the question of the effect of magicity on nuclear incompressibility. Despite a specific prediction [11], no evidence of the mutual magicity enhancement (MEM) effect for the incompressibility was found experimentally [13]. The absence of the MEM effect was further confirmed by additional studies [6,12]. However, 


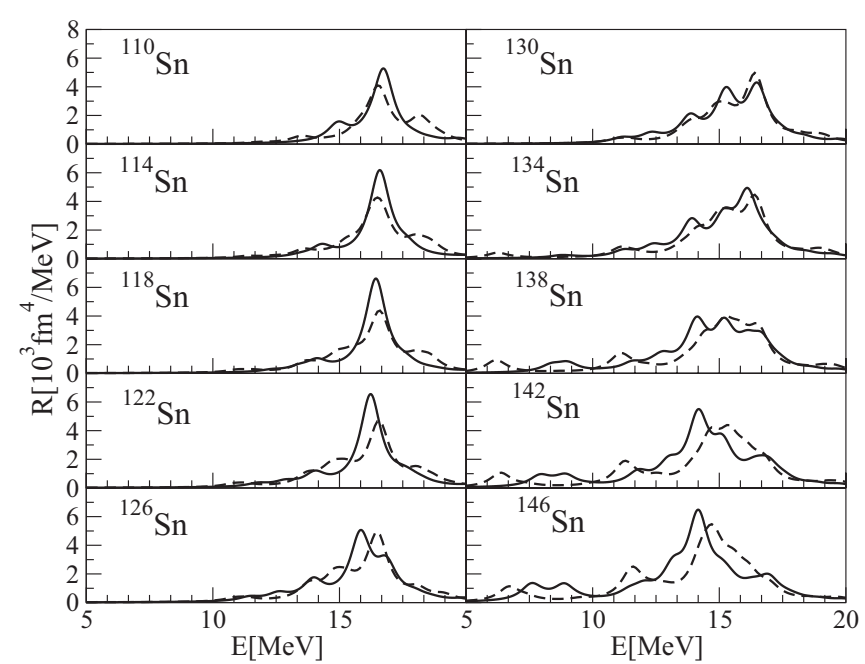

FIG. 2. Isoscalar monopole response in Sn isotopes, calculated using the relativistic QRPA with the DD-ME2 functional (solid) and the QRPA with the functional SLy5 (dashed). The response is folded with a Lorentzian of $1 \mathrm{MeV}$ width.

the present results show that just beyond major-shell closure nuclear incompressibility decreases because of the appearance of the soft monopole mode. One could, therefore, conclude that a magicity effect on the incompressibility is predicted, although it is not the MEM effect.

Figures 2 and 3 clearly exhibit the emergence of a soft monopole mode in the QRPA monopole response functions of neutron-rich nuclei. It might, therefore, be useful to calculate the values of the incompressibility $K_{A}$ separately in the lowenergy region of the soft mode and in the energy region of the GMR. The results are shown in Figs. 4 and 5, where the isotopic dependence of this values, calculated using the QRPA with the functional SLy5, is plotted for a series of $\mathrm{Sn}$ and $\mathrm{Pb}$ nuclei, respectively. The boundary between the low-energy and GMR regions is set arbitrarily at $13.5 \mathrm{MeV}$ for $\mathrm{Sn}$ isotopes and at $11 \mathrm{MeV}$ for $\mathrm{Pb}$ isotopes. $K_{\text {soft }}\left(K_{\mathrm{GMR}}\right)$ is determined

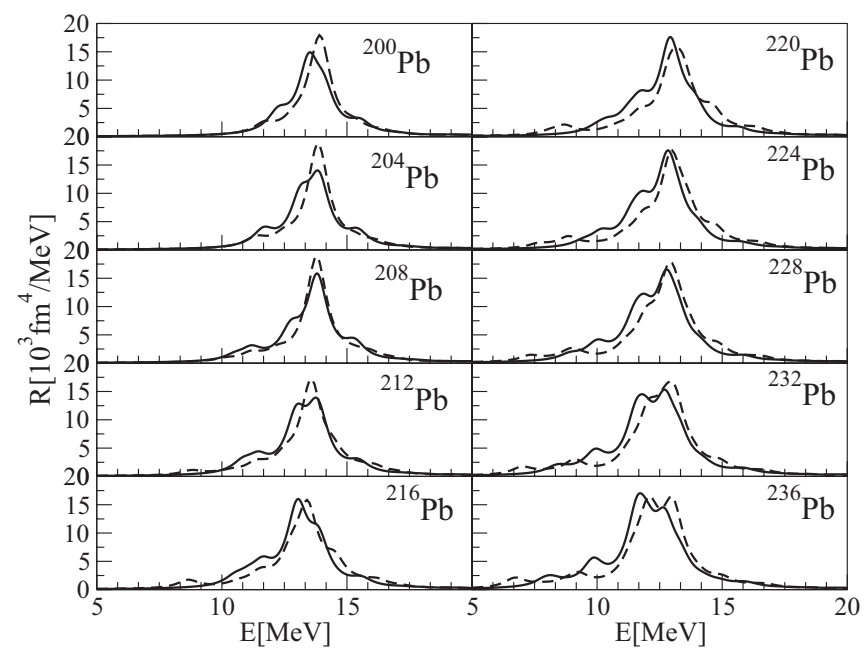

FIG. 3. Same as described in the caption to Fig. 2 but for the $\mathrm{Pb}$ isotopes.

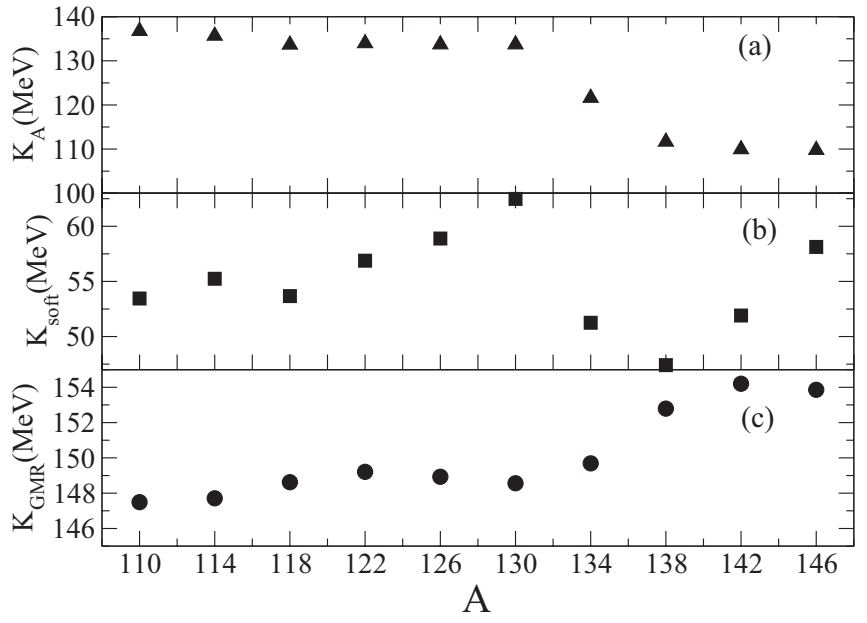

FIG. 4. Nuclear incompressibility in the Sn isotopic chain calculated using the microscopic Skyrme-QRPA with the functional SLy5. (a) Total nuclear incompressibility $K_{A}$. (b) Values determined by the monopole response in the low-energy region. (c) Values for the GMR region.

from the monopole response below (above) this threshold. The isotopic dependence of $K_{\text {soft }}$ and $K_{\mathrm{GMR}}$ reflects the evolution of the soft monopole mode and the GMR. While in the case of the $\mathrm{Sn}$ isotopes no definite trend is found, for the $\mathrm{Pb}$ nuclei both $K_{\text {soft }}$ and $K_{\mathrm{GMR}}$ show a steady decrease with increasing neutron number.

\section{CONCLUSIONS}

The incompressibility of finite fermionic systems has been studied using several analytical and numerical approaches. Analytic relations obtained using a simple free Fermi gas or harmonic oscillator model point to a direct link between the incompressibility of the system and the zero-point kinetic energy of constituent particles. The incompressibility is thus related to the localization of the individual fermions. For

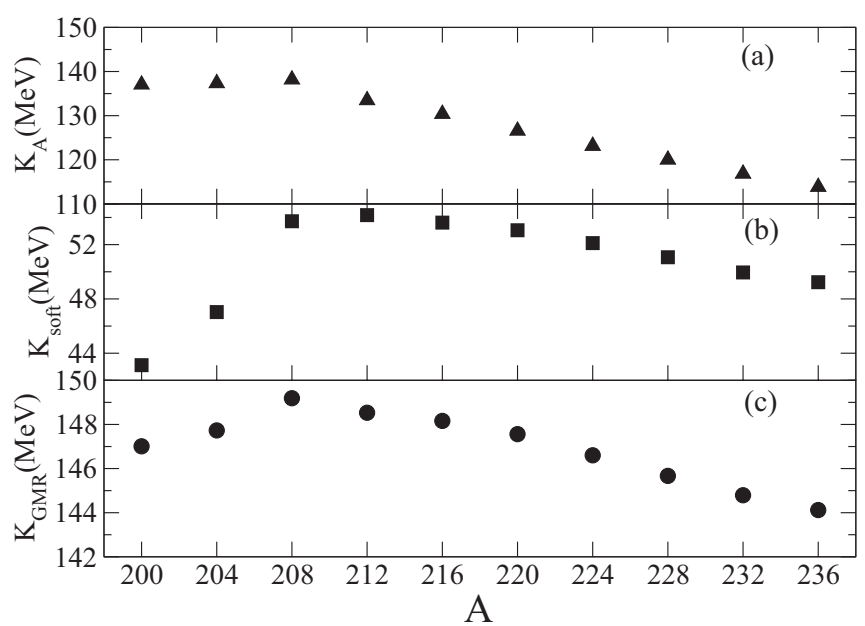

FIG. 5. Same as described in the caption to Fig. 4 but for the $\mathrm{Pb}$ isotopes. 
atomic nuclei the $\mathrm{HO}$ model predicts the incompressibility $K_{A} \approx 5 T_{0} \approx 140 \mathrm{MeV}$.

To quantitatively analyze the evolution of nuclear incompressibility in isotopic chains of medium-heavy and heavy nuclei, microscopic EDF-based approaches have been used: the constrained HFB method and the QRPA based on both nonrelativistic and relativistic density functionals. All microscopic models predict a remarkably similar trend for the incompressibility: an almost constant behavior in stable nuclei and systems not far from stability, with values of $K_{A}$ close to those predicted by the HO model, and a steep decrease of $K_{A}$ in nuclei with pronounced neutron excess, caused by the emergence of a soft monopole mode in neutronrich nuclei. It would be important to have experimental confirmation of the soft monopole mode in neutron-rich nuclei and, more generally, to study the behavior of the monopole response in exotic nuclei and/or beyond shell closures. For instance, measurement of the GMR beyond ${ }^{132} \mathrm{Sn}$ and ${ }^{208} \mathrm{~Pb}$ would be very interesting.

\section{ACKNOWLEDGMENTS}

This work was supported by the Institut Universitaire de France and by MZOS Project No. 1191005-1010.
[1] T. Li et al., Phys. Rev. Lett. 99, 162503 (2007).

[2] T. Li et al., Phys. Rev. C 81, 034309 (2010).

[3] D. Patel et al., Phys. Lett. B 718, 447 (2012).

[4] J. Li, G. Colò, and J. Meng, Phys. Rev. C 78, 064304 (2008).

[5] E. Khan, Phys. Rev. C 80, 011307(R) (2009).

[6] P. Veselý, J. Toivanen, B. G. Carlsson, J. Dobaczewski, N. Michel, and A. Pastore, Phys. Rev. C 86, 024303 (2012).

[7] O. Sorlin and M.-G. Porquet, Prog. Part. Nucl. Phys. 61, 602 (2008).

[8] E. Khan, J. Margueron, and I. Vidaña, Phys. Rev. Lett. 109, 092501 (2012).

[9] C. Monrozeau et al., Phys. Rev. Lett. 100, 042501 (2008).

[10] M. Vandebrouck et al., Exp. E456a performed at GANIL (2010).

[11] E. Khan, Phys. Rev. C 80, 057302 (2009).

[12] L.-G. Cao, H. Sagawa, and G. Colò, Phys. Rev. C 86, 054313 (2012).

[13] U. Garg (private communication).

[14] S. Shlomo, V. M. Kolomietz, and G. Colò, Eur. Phys. J. A 30, 23 (2006).

[15] M. Bender, P.-H. Heenen, and P.-G. Reinhard, Rev. Mod. Phys. 75, 121 (2003).

[16] D. Vretenar, A. V. Afanasjev, G. A. Lalazissis, and P. Ring, Phys. Rep. 409, 101 (2005).

[17] J.-P. Blaizot, Phys. Rep. 64, 171 (1980).

[18] O. Bohigas, A. M. Lane, and J. Martorell, Phys. Rep. 51, 267 (1979).

[19] I. Hamamoto, H. Sagawa, and X. Z. Zhang, Phys. Rev. C 53, 765 (1996).

[20] L. Capelli, G. Colò, and J. Li, Phys. Rev. C 79, 054329 (2009).
[21] E. Khan, N. Paar, and D. Vretenar, Phys. Rev. C 84, 051301(R) (2011)

[22] L. Landau and E. Lifchitz, Statistical Physics (MIR Edition, Moscow, 1967).

[23] D. J. Thouless, Nucl. Phys. 22, 78 (1961).

[24] A. L. Fetter and J. D. Walecka, Quantum Theory of ManyParticle Systems (McGraw-Hill, New York, 1971).

[25] B. Mottelson, Trends in Nuclear Physics, 100 Years Later, UJFG, Les Houches, session LXVI (Elsevier, Amsterdam, 1996), p. 25.

[26] M. Harakeh and A. Van der Woude, Giant Resonances (Oxford University Press, New York, 2001).

[27] N. Paar, D. Vretenar, E. Khan, and G. Colò, Rep. Prog. Phys. 70, 691 (2007).

[28] E. Chabanat, P. Bonche, P. Haensel, J. Meyer, and R. Schaeffer, Nucl. Phys. A 635, 231 (1998).

[29] E. Khan, M. Grasso, and J. Margueron, Phys. Rev. C 80, 044328 (2009).

[30] P. Ring and P. Schuck, The Nuclear Many-Body Problem (Springer-Verlag, Heidelberg, 1980).

[31] G. Colò, L. G. Cao, N. Van Giai, and L. Capelli, Comput. Phys. Commun. 184, 142 (2013).

[32] T. Nikšić, D. Vretenar, P. Finelli, and P. Ring, Phys. Rev. C 66, 024306 (2002).

[33] N. Paar, P. Ring, T. Nikšić, and D. Vretenar, Phys. Rev. C 67, 034312 (2003).

[34] J. F. Berger, M. Girod, and D. Gogny, Comput. Phys. Commun. 63, 365 (1991).

[35] G. A. Lalazissis, T. Nikšić, D. Vretenar, and P. Ring, Phys. Rev. C 71, 024312 (2005). 